\title{
28 Research Square \\ The oviposition cue indole inhibits animal-host attraction in Aedes aegypti mosquitoes
}

\section{Amir Dekel}

The Hebrew University of Jerusalem

\section{Evyatar Sar-Shalom}

The Hebrew University of Jerusalem

\section{Esther Yakir}

The Hebrew University of Jerusalem

Jonathan Daniel Bohbot ( $\boldsymbol{\sim}$ jonathan.bohbot@mail.huji.ac.il )

Hebrew University of Jerusalem Robert H Smith Faculty of Agriculture Food and Environment https://orcid.org/0000-0001-5204-6340

\section{Research Article}

Keywords: Aedes aegypti, indole, DEET, IR3535, (R)-1-octen-3-ol, OR8, repellent

Posted Date: May 4th, 2021

DOI: https://doi.org/10.21203/rs.3.rs-418857/v1

License: (c) (1) This work is licensed under a Creative Commons Attribution 4.0 International License. Read Full License 


\section{Abstract}

Mosquitoes represent a major source of disease transmission and possess the uncanny ability to locate suitable animal-hosts, a feature mediated by their exquisite olfactory system. Insect repellents such as $\mathrm{N}, \mathrm{N}$-Diethyl-meta-toluamide, also called DEET, have been shown to activate and inhibit mosquito odorant receptors resulting in behavioral modulation. This and other repellents available for personal protection against mosquitoes are topically applied on the skin and operate at short range. In our search for potential long-range odorant repellents, we have hypothesized that the shared chemical similarities between indole and DEET, may confer the former the ability to block odorant receptor function and inhibit human-host attraction. Using the two-electrode voltage clamp of Xenopus laevis oocytes as a pharmacological platform, we provide evidence that indole inhibits the Aedes aegypti $(R)$-1-octen-3-ol receptor OR8, a receptor involved in the decision of female mosquitoes to identify human hosts. Coincidentally, we also show that indole inhibits the animal-host seeking behavior of female Aedes aegypti. Together, our findings suggests that indole may be a candidate spatial repellent for the longrange protection of humans against mosquito bites.

\section{Full Text}

This preprint is available for download as a PDF.

\section{Figures}


A

(R)-1-octen-3-ol

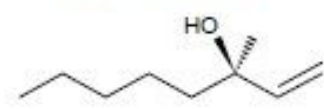<smiles>COc1cccc2[nH]ccc12</smiles><smiles>CCN(CC)C(=O)c1cccc(I)c1</smiles>

B
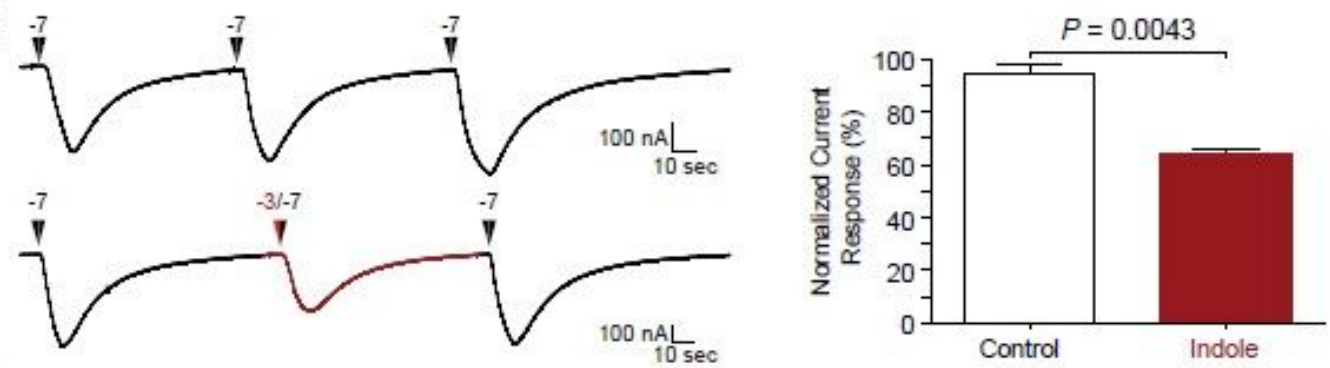

C
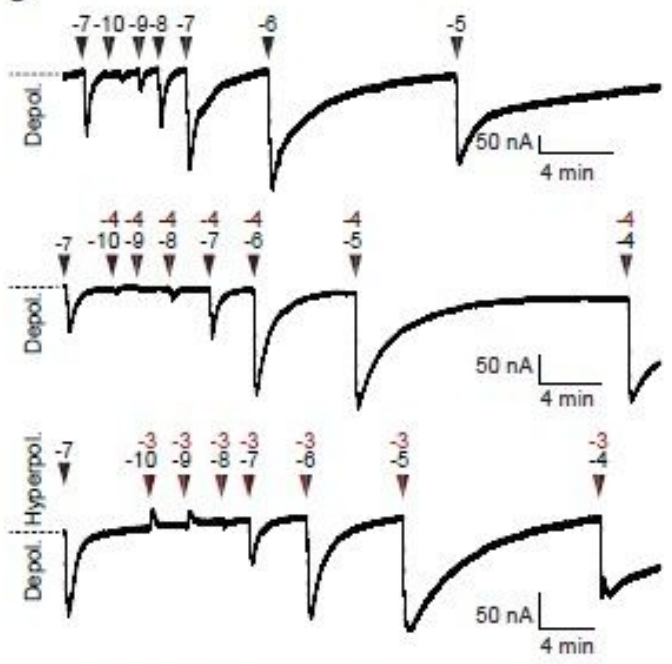

D
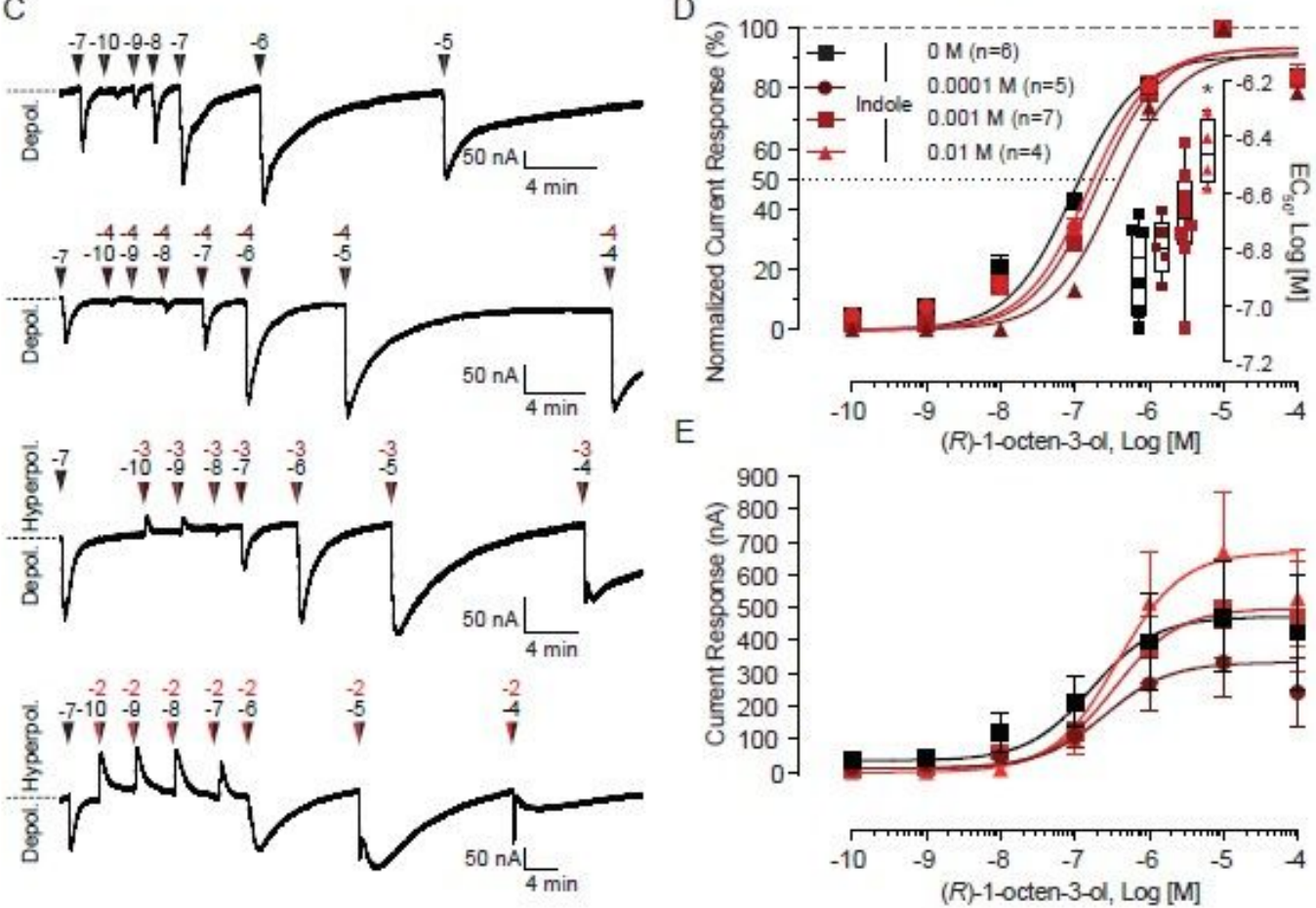

$\mathrm{F}$
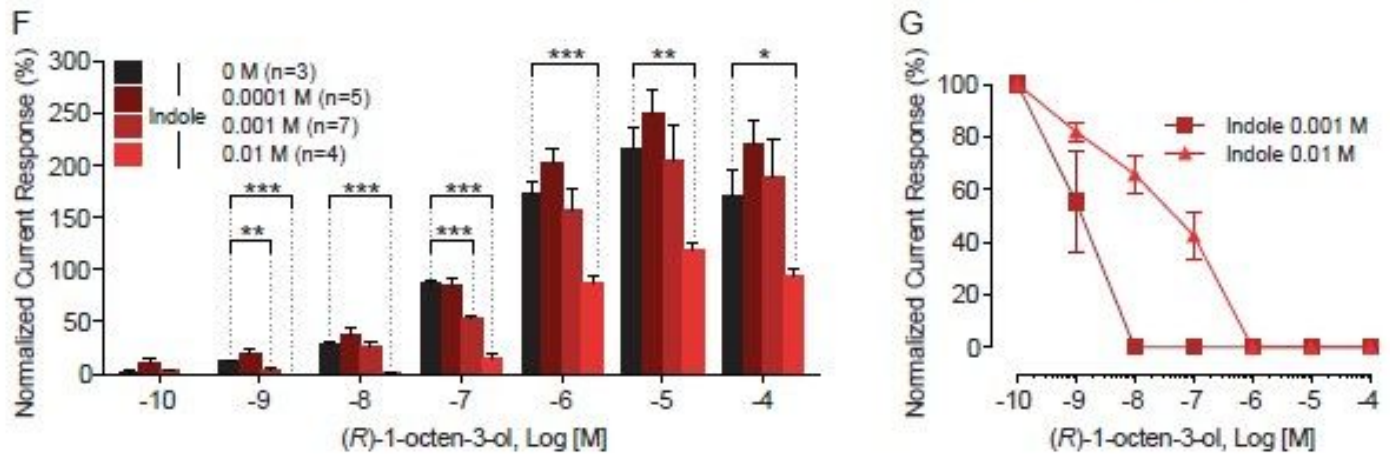

\section{Figure 1}

Indole inhibits OR8-Orco activation by (R)-1-octen-3-ol. A) (R)-1-octen-3-ol is an alkenyl alcohol and a potent ligand of OR8. Indole is an aromatic bicyclic organic compound. N,N-Diethyl-meta-toluamide (DEET) is a benzyl-ester sharing a benzene ring and a nitrogen atom with indole (red features). B) Representative current traces of AaegOR8-Orco activation by 10-7 M (R)-1-octen-3-ol (black arrowheads) or by a mixture of 10-3 M indole (red arrowhead) and 10-7 M (R)-1-octen-3-ol (black arrowhead). 
Normalized responses of AaegOR8-Orco to 10-7 M (R)-1-octen-3-ol alone (control) or in combination with 10-3 $\mathrm{M}$ indole (indole). Statistical significance was determined using the Wilcoxon-Mann-Whitney test $(n=5-6)$. C) Representative current traces of AaegOR8-Orco activation by (R)-1-octen-3-ol alone or in the presence of 10-4 M, 10-3 M or 10-2 $\mathrm{M}$ indole. Arrowheads above the traces indicate the onset of the odorant stimulus, black arrowheads represent (R)-1-octen-3-ol and red arrowheads represent indole. D) Concentration-response relationships of AaegOR8-Orco in response to increasing indole concentrations. Current amplitudes were normalized to the maximum response. Statistical significance was determined using a One-Way ANOVA $(P=0.0142)$ followed by a Dunn's multiple comparisons test $(n=4-7)$. E) Nonnormalized concentration current response relationships $(n=4-7)$. F) Histogram of the depolarization current response amplitudes of AaegOR8-Orco in response to (R)-1-octen-3-ol alone (black) or in combination with various indole concentrations (shades of red). Statistical significance was determined using multiple t-test and statistical significance determined by the Holm-Sidak method ( $*$ : $\leq 0.05$; $* *$ : $\leq 0.01 ; * * *: P \leq 0.001 ; n=3-7) . G$ ) Concentration-response relationships of indole induced hyperpolarization currents exhibited by AaegOR8-Orco-injected oocytes. 

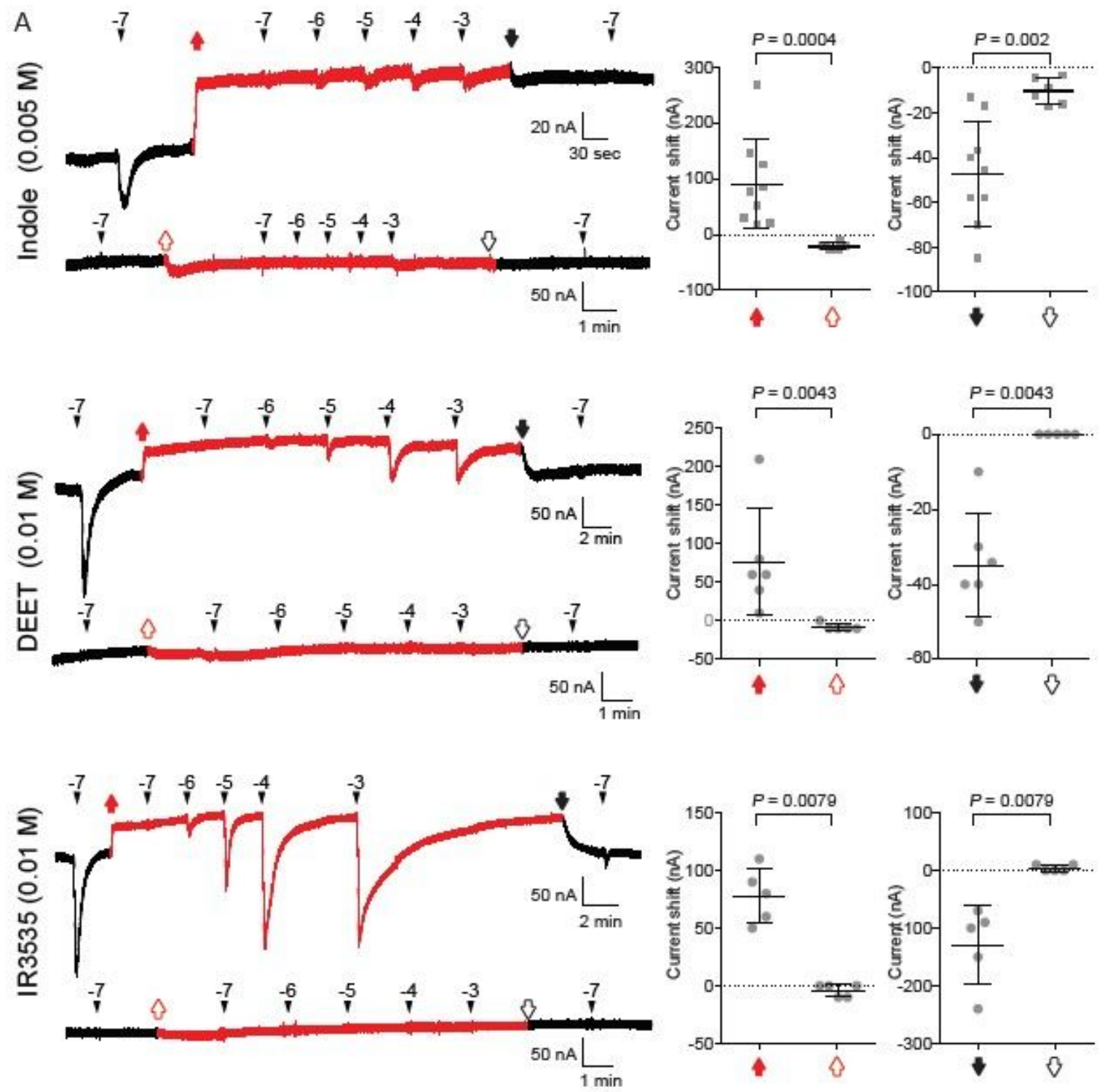

B ND96 $(R)$-1-octen-3-ol Indole

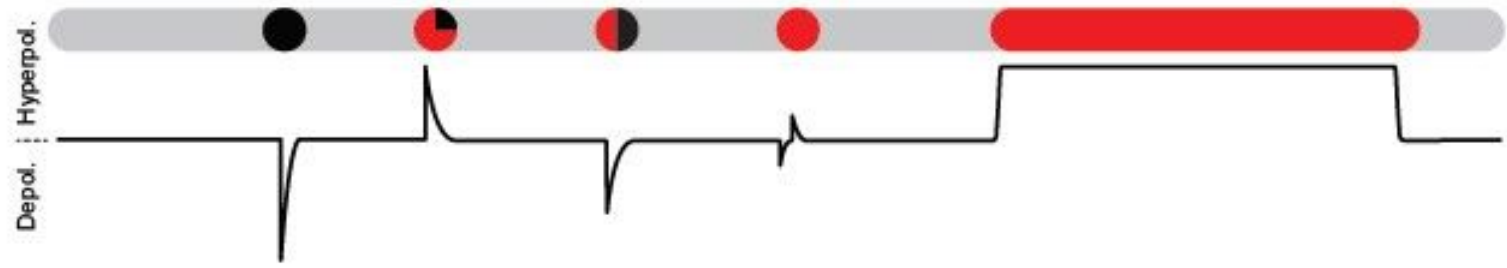

\section{Figure 2}

High indole concentrations elicit both depolarization and hyperpolarization currents. A) Representative current traces of AaegOR8-Orco activation by $8 \mathrm{sec}$ stimulations of (R)-1-octen-3-ol alone or increasing indole concentrations (10-6-10-2 M). Arrowheads above the traces indicate the onset of the odorant stimulus, black arrowheads represent (R)-1-octen-3-ol and red arrowheads represent indole. $B$ ) Representative current trace of AaegOR8-Orco activation by 10-7 M (R)-1-octen-3-ol (black arrowhead) 
and by 10-2 $\mathrm{M}$ indole (red arrowhead). Statistical significance was determined using the WilcoxonMann-Whitney test $(n=7)$. C) (R)-1-Octen-3-ol is required to elicit significant indole-induced hyperpolarization currents. Nanomolar concentrations of (R)-1-octen-3-ol induce larger indole induced depolarization currents than indole alone. Representative traces are shown above. Statistical significance was determined using a t-test $(n=7-12)$.
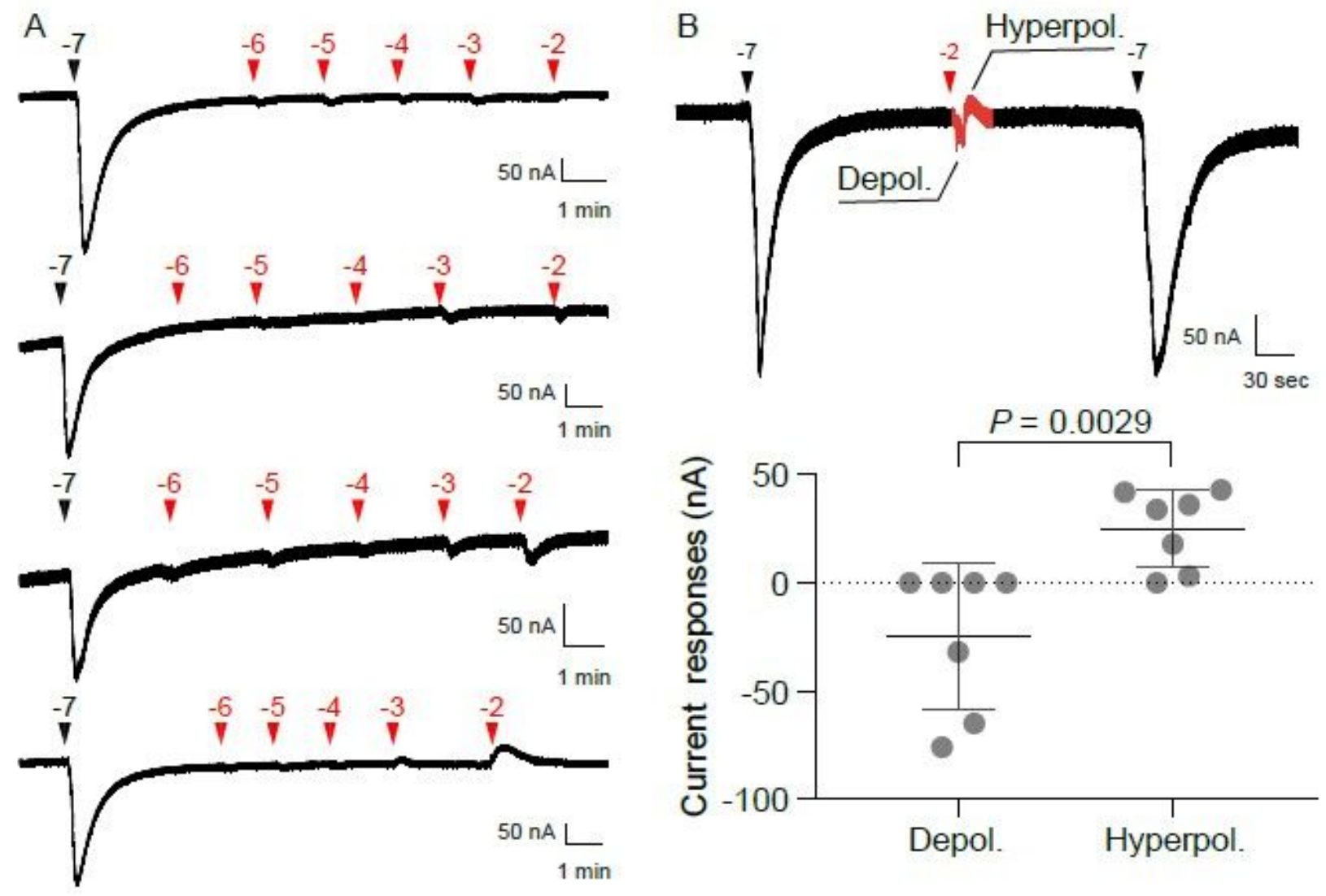

$\mathrm{C}$
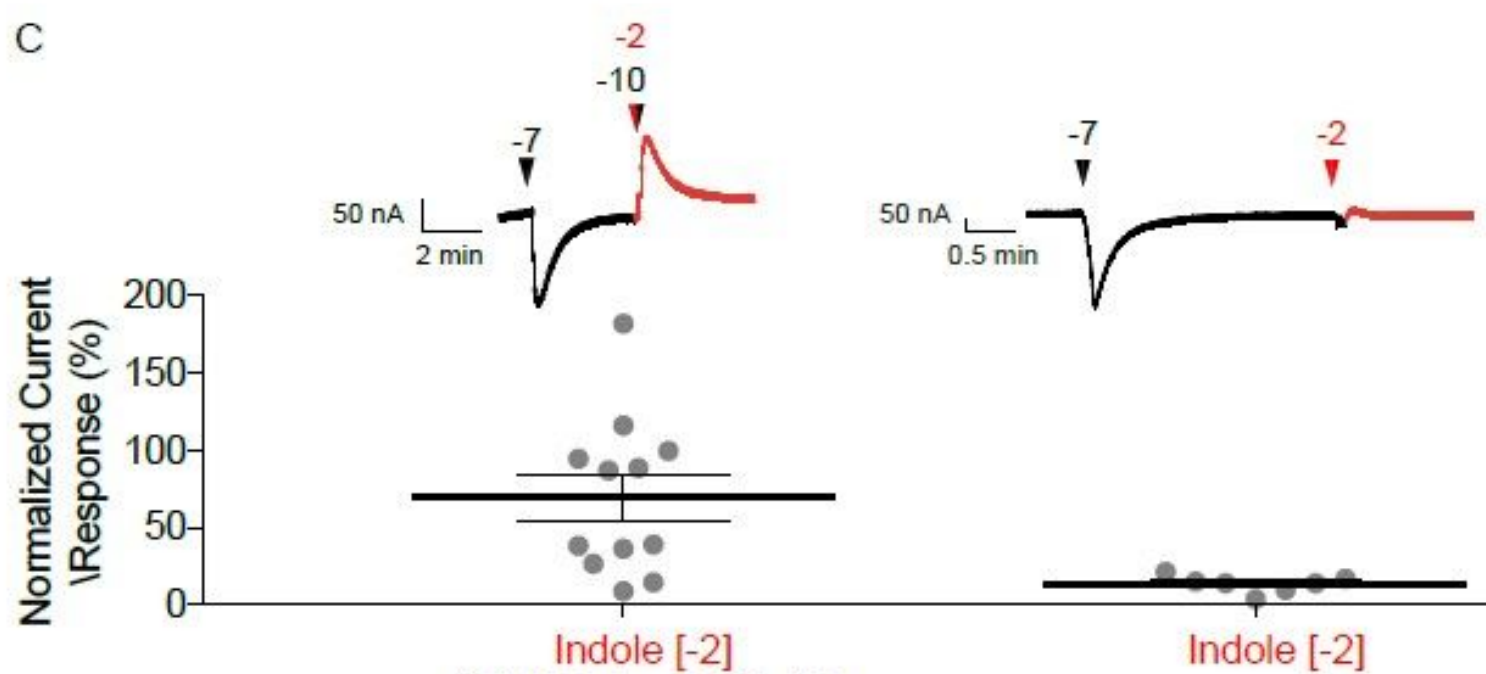

$(R)-1$-Octen-3-ol [-10]

$P=0.0037$

Figure 3 
Indole and insect repellents increase the baseline current state of AaegOR8-Orco-injected oocytes. Representative current traces of AaegOR8-Orco successively exposed to $100 \mathrm{nM}$ (R)-1-octen-3-ol under ND96 (black trace), continuous perfusion under indole (0.005 M), DEET (0.01 M), and IR3535 (0.01 M) perfusions (red traces) with increasing concentrations of (R)-1-octen-3-ol, ND96 perfusion, and $100 \mathrm{nM}$ (R)-1-octen-3-ol. The same protocol was applied to water-injected oocytes as well. In AaegOR8-Orcoinjected oocytes, perfusion buffer transitions from ND96 to indole, DEET or IR3535 are shown as solid red arrows, while the reverse perfusion transitions are shown as a solid black arrow. In water-injected oocytes, the corresponding buffer transitions are indicated by empty red and black arrows. The baseline currents of OR8-Orco-injected oocytes exhibit significant increases and decreases compared to water-injected oocytes. Statistical significance was determined using the Wilcoxon-Mann-Whitney test $(n=5-9)$. B) Under saline perfusion (ND96), AaegOR8-Orco elicits a stable current baseline. A stimulus pulse of (R)- 1octen-3-ol activates AaegOR8-Orco, which translates into a transient whole cell depolarization current. A pulse of (R)-1-octen-3-ol (low concentration) and indole (high concentration) mixture transiently induces a hyperpolarization current. Increasing (R)-1-octen-3-ol concentrations reverses indole blockade. High indole concentrations elicit small depolarization and hyperpolarization currents. Under indole perfusion, the current baseline is increased. 

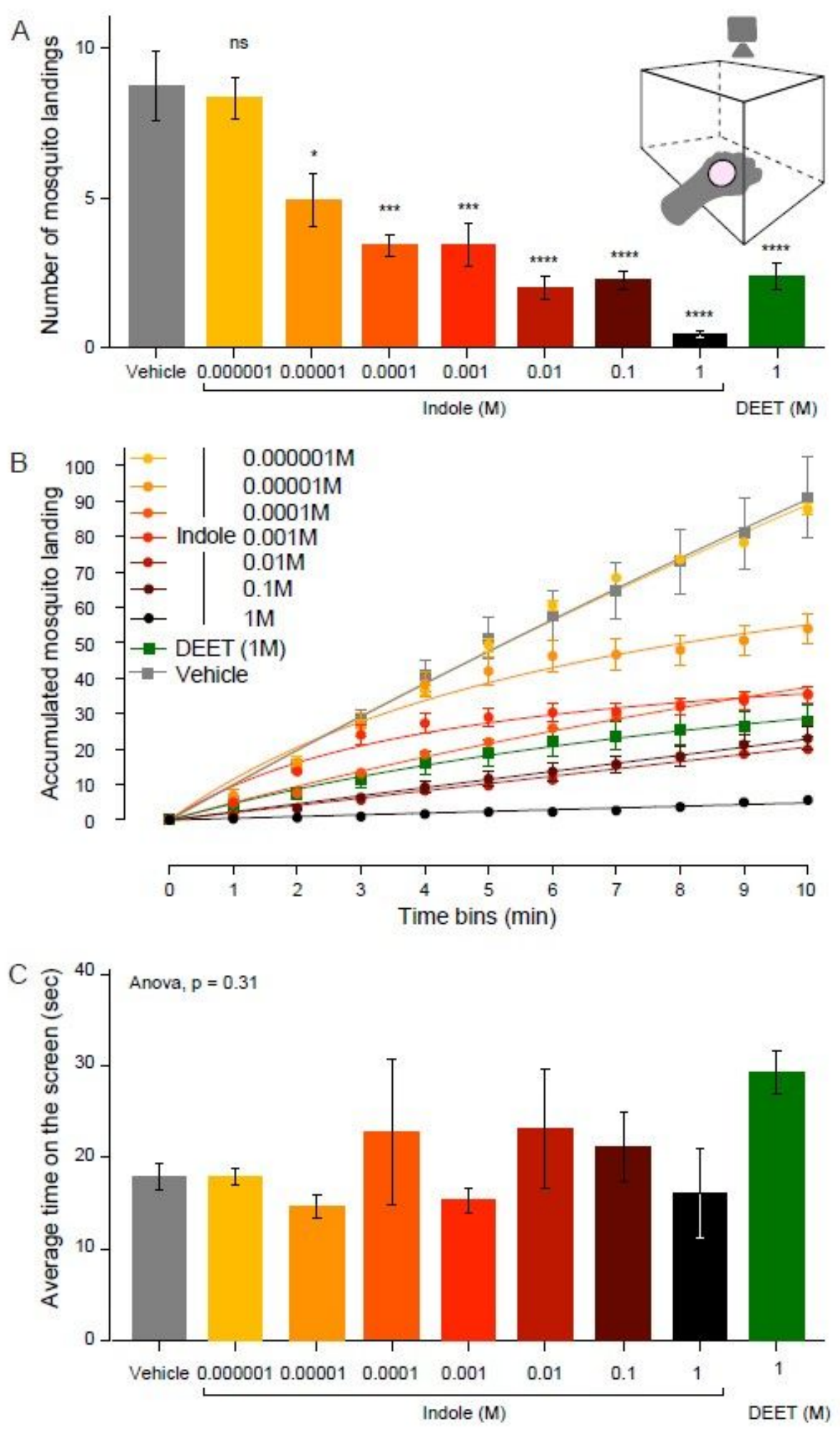

Figure 4

Indole repels human-seeking female Ae. aegypti mosquitoes. A) Diagram of the arm-in-cage assay (inset). The gloved experimenter's hand is introduced inside a cage containing 15 Ae. aegypti female mosquitoes. The exposed skin area was monitored by a recording camera. The number of mosquito visits were counted as a function of treatments, including diethyl ether (vehicle), DEET $1 \mathrm{M}$ and 7 indole concentrations. All treatment groups were analyzed compared to vehicle using a Wilcoxon rank-sum test 
$(n=3)$. B) Hyperbola line fitting of the cumulative number of mosquito landings over time. Points represented are mean \pm SEM $(n=3)$. See accompanying supplementary figure 2 . C) Histogram of the average time duration mosquitoes spent on the exposed skin area. A one-way ANOVA analysis with Tukey multiple comparisons of means $95 \%$ family-wise confidence level was used to analyze all treatment groups compared to control (vehicle). Shapiro-Wilk normality test ( $W=0.95056, P=0.2212$ ) was performed on the square root of the dependent variable $(n=3)$. 